\title{
Green production of biodegradable mulch films for effective weed control
}

Ke Wang ${ }^{* 1,2}$ Xiaoyan Sun ${ }^{1}$, Bibo Long ${ }^{1}$, Fayong Li ${ }^{1}$, Chong Yang ${ }^{1}$, Junjia Chen ${ }^{1}$, Chunping $\mathrm{Ma}^{3}$, Dong Xie ${ }^{* 1}$, and Yen Wei ${ }^{* 2}$

1 Institute of Bioengineering and Guangdong Biomaterials Engineering Technology Research Center, Guangdong Academy of Sciences, Guangzhou, 510316, China

2 Department of Chemistry and the Tsinghua Center for Frontier Polymer Research, Tsinghua University, Beijing, 100084, China

3 Guangdong-HongKong Joint Laboratory for New Textile Materials, School of Textile Materials and Engineering, Wuyi University, Jiangmen, 529020, China 


\section{Contents}

Figure S1

Figure S2

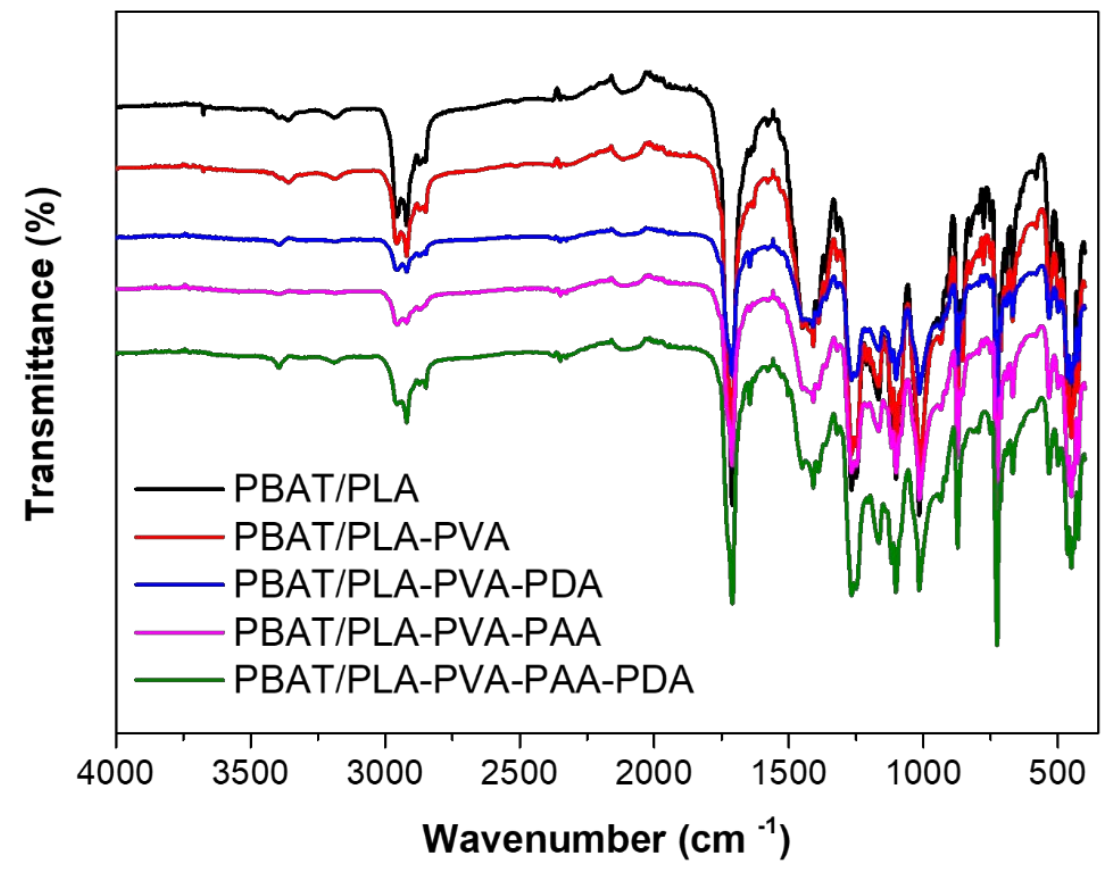

Figure S1. FTIR spectra of films with different coatings. 


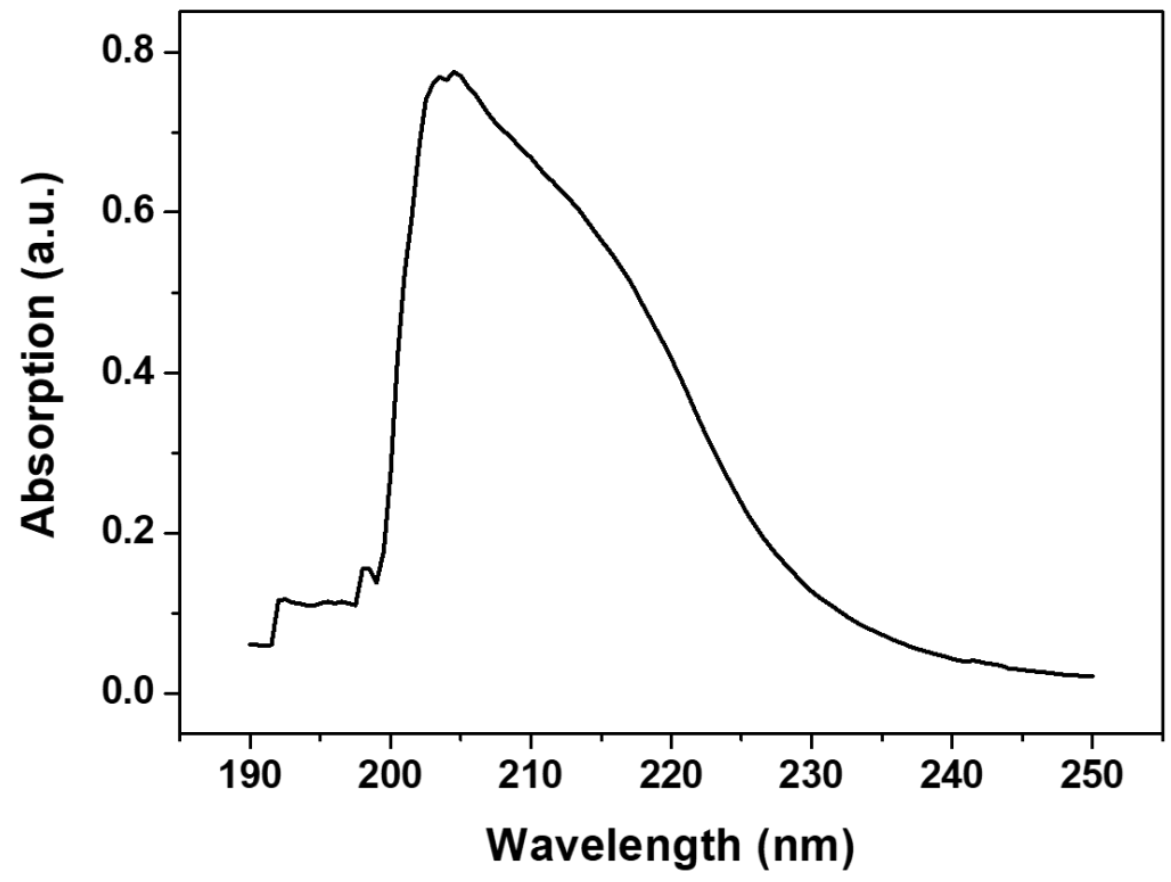

Figure S2. The absorption curve of acetochlor. 